Cahiers de littérature orale

75-76 | 2014

L'autre voix de la littérature

\title{
Trouver les mots justes
}

Échos d'un témoignage écrit sur les années 1950 en Amdo (Tibet)

\section{Xénia de Heering}

\section{OpenEdition}

\section{Journals}

Édition électronique

URL : https://journals.openedition.org/clo/2030

DOI : $10.4000 /$ clo.2030

ISSN : 2266-1816

Éditeur

INALCO

Édition imprimée

Date de publication : 1 janvier 2014

ISBN : 978-2-85831-222-1

ISSN : 0396-891X

Référence électronique

Xénia de Heering, "Trouver les mots justes », Cahiers de littérature orale [En ligne], 75-76 | 2014, mis en ligne le 18 mai 2015, consulté le 01 juillet 2021. URL : http://journals.openedition.org/clo/2030 ; DOI : https://doi.org/10.4000/clo.2030

Ce document a été généré automatiquement le 1 juillet 2021

\section{(c) (7) \&)}

Cahiers de littérature orale est mis à disposition selon les termes de la Licence Creative Commons Attribution - Pas d'Utilisation Commerciale 4.0 International. 


\title{
Trouver les mots justes
}

\author{
Échos d'un témoignage écrit sur les années 1950 en Amdo (Tibet)
}

\section{Xénia de Heering}

« Raconter bien, ça veut dire : de façon à être entendus »

Jorge Semprun $(1994,165)$

\section{Introduction}

1 Le livre Joies et peines de l'enfant Naktsang (Nags tshang zhi lu'i skyid sdug) ${ }^{1}$ a été publié en 2007 à Xining, capitale de la province du Qinghai, en République populaire de Chine (RPC). Ce témoignage est l'œuvre non d'un écrivain professionnel, mais d'un fonctionnaire tibétain à la retraite, originaire de Chukhama. Cette localité, aujourd'hui incluse dans la Préfecture autonome tibétaine (PAT) du Kanlho (chi. : Gannan), dans la province du Gansu, appartient à la région de l'Amdo, qui occupe le nord-est du Plateau tibétain ${ }^{2}$. Le récit autobiographique de Naktsang Nülo, écrit à la première personne, débute avec sa naissance en 1948 et s'achève lors de son entrée à l'école, le 30 décembre 1959. L'auteur y évoque des pratiques et un environnement familiers - la vie sous la tente, les activités des pasteurs, les jeux des enfants, les relations familiales, les pratiques religieuses, etc. - puis leur bouleversement complet en 1958, année de changements radicaux, de révoltes, de répression armée et de violences sans précédent exercées à l'encontre des civils (Robin, 2012), qui verra aussi le début de la famine qui frappa l'ensemble de la RPC à cette époque, suite au lancement du Grand Bond en avant.

2 Joies et peines de l'enfant Naktsang est un des premiers ouvrages publiés en RPC - et le premier à avoir connu une telle diffusion - à raconter en détail l'expérience de ces événements, avec la particularité de le faire depuis le point de vue de l'enfant de dix ans qui ne connaissait pas encore l'issue du "grand renversement d'époque » (dus 'gyur chen mo) survenu cette année-là en Amdo. On peut imaginer l'émotion et l'écho suscités par ce récit, sans précédent, d'événements traumatiques qui hantent les esprits, mais demeurent à ce jour bannis de l'espace public par le gouvernement central à Pékin. Alors que le tirage habituel des livres en tibétain dépasse rarement deux mille 
exemplaires, le tirage cumulé de Joies et peines, grâce aux nombreuses contrefaçons dont l'ouvrage a très vite fait l'objet, auraient atteint quarante à cinquante mille exemplaires ${ }^{3}$. On peut supposer, dans ces conditions, que les lecteurs en appréciaient tant la valeur historique que la qualité narrative.

3 Cet ouvrage constitue une forme particulière de narration historique, à bien des égards novatrice. Au cours de l'enquête, la langue employée dans le récit est d'emblée apparue comme un critère de description saillant, donnant lieu à des réactions et à des commentaires très contrastés ${ }^{4}$. On me décrivait souvent Joies et peines comme un livre "écrit en kha skad (langue orale)", le réduisant parfois même à cette seule caractéristique. En s'appuyant sur une ethnographie des réceptions de Joies et peines, cette étude analyse la diversité des appréciations formulées à l'égard des langues orales dans les évaluations de ce témoignage et de sa langue très inhabituelle au regard des pratiques écrites dominantes.

4 Si la nouveauté, la signification sociale et la portée historique de Joies et peines dépassent largement la question de la langue, placer celle-ci au cœur de l'analyse permet d'apporter des éléments décisifs pour décrire la pluralité des réceptions de l'ouvrage, mais aussi pour rendre compte de l'importance de sa diffusion. Décrire des expériences de lecture, inobservables directement, expériences d'interprétation variant à l'infini selon les biographies et les bibliothèques de chaque lecteur, constitue un exercice délicat. Les témoignages épars, parfois allusifs, toujours situés, d'un "échantillon" de lecteurs très restreint au regard du lectorat total, seront ici contextualisés et comparés, dans la perspective d'identifier les critères mobilisés par des lecteurs qui, nombreux en Amdo, reconnaissent à l'enfant Naktsang une certaine justesse de ton.

\section{Réceptions contrastées d'un texte " écrit en kha skad »}

5 «À chaque vallée son dialecte, à chaque lama sa religion " (lung pa re la skad lugs re, bla ma re la chos lugs re). Ce proverbe, dont il existe des variantes dans toutes les régions tibétophones, témoigne d'une reconnaissance interne et omniprésente de la diversité linguistique. Sa diffusion constitue également un indice des étroites relations culturelles, religieuses et commerciales historiquement entretenues par ces différentes régions en dépit du morcellement politique qui a prévalu depuis la fin de l'Empire tibétain (VII-IX ${ }^{\text {es }}$ siècles).

6 Les réceptions de Joies et peines, et les appréciations de sa langue, ne peuvent être saisies que dans le contexte du système écolinguistique complexe où l'ouvrage est reçu. L'aire linguistique tibétaine, qui s'étend sur environ $2400000 \mathrm{~km}^{2}$, à cheval entre six pays (RPC, Inde, Népal, Bhoutan, Pakistan et Birmanie), se présente comme un enchevêtrement dans lequel les linguistes distinguent près de deux cents dialectes. Certains dialectes, dont celui de l'Amdo, "sont si différents et permettent si peu d'intercompréhension que l'on serait en droit de parler de langues distinctes », et distinguer selon ce critère une dizaine de langues au sein de l'aire linguistique tibétaine (Tournadre, 2005, 17). La communication orale est ainsi limitée, voire impossible, entre locuteurs de l'Amdo, de l'Ü-tsang (Tibet central) et du Kham (Tibet oriental), les trois entités constitutives du « Tibet aux trois régions » (Bod chol ka gsum). 
7 L'ensemble de ces dialectes, "étroitement liés au vieux tibétain», utilise traditionnellement une même langue écrite, le tibétain littéraire, dont la forme moderne demeure assez proche de la langue littéraire classique (Tournadre, 2005, 16). Par-delà la profusion des dialectes, cette culture écrite commune constitue l'un des aspects les plus saillants de la cohésion de l'aire culturelle tibétaine. En dépit du fait que de nombreux termes dialectaux possèdent une étymologie écrite, il est courant d'opposer le tibétain littéraire, ou langue écrite (bod yig, yig skad), aux langues parlées ou orales (kha skad), comme deux domaines bien distincts. Les jugements exprimés au sujet de Joies et peines mobilisent ainsi des normes et des idées quant à ce qui constitue une expression écrite correcte ou esthétique, mais aussi des aspirations quant à l'avenir et à l'unification de la langue tibétaine, au regard desquelles on redoute les conséquences néfastes de l'écriture en langue régionale. L'écrit et l'oral ne peuvent cependant pas être envisagés comme des entités déconnectées, hermétiques ou figées. Des usages de l'écrit plus proches de l'oralité semblent notamment favorisés par l'apparition de nouveaux supports : on voit ainsi couramment du kha skad écrit sur les forums en ligne ou dans les sms, mais il est rare qu'un texte imprimé n'obéisse pas aux normes de la langue littéraire.

26 mai 2009, Paris, entretien avec Noyontsang Lhamokyap, originaire de la PAT de Malho (chi. : Huangnan, Qinghai), à quelques deux cents kilomètres de Chukhama:

[Dans Joies et peines,] certains disent que la manière de parler produit une vision de ces gens - ce sont des pasteurs ('brog pa) de l'Amdo, et des gens de Machu. On est persuadé que c'est un vieux de Chukhama, à Machu, à partir de la manière de parler, on le voit vraiment. Ce genre d'impression, d'image, elle vient vraiment de la manière de parler.

8 Réaliste dans sa représentation de la langue parlée, l'écriture de Nülo constitue aussi une économie de la description. "On est persuadé que c'est un vieux de Chukhama, à Machu, à partir de la manière de parler, on le voit vraiment. " L'intercompréhension parfois laborieuse entre locuteurs de différentes régions n'est en effet qu'une des conséquences pratiques de la différenciation dialectale. Une autre est la possibilité de reconnaître l'origine des locuteurs « dès qu'un inconnu se met à parler " - comme l'indique Nicolas Tournadre, «certaines personnes ayant voyagé dans l'aire linguistique sont expertes à ce jeu et peuvent immédiatement déceler avec précision la provenance des personnes » (Tournadre, 2005, 17). Nülo n'a nul besoin, dans son texte, de préciser l'origine de ceux qu'il nous donne à entendre ni de décrire leur apparence physique et vestimentaire. Il lui suffit, dans le discours direct, de transcrire leur manière de parler pour que le lecteur puisse immédiatement s'en faire une image.

17 octobre 2011, Dharamsala, homme d'environ 40 ans, originaire de Labrang (chi. : Xiahe, PAT du Kanlho), à environ trois cents kilomètres de Chukhama :

Il y a beaucoup de fautes... En fait ce ne sont pas des fautes, mais certains mots que l'auteur met par écrit, qui n'ont pas d'orthographe, comme la manière dont les mères appellent leurs enfants dans cette région-là, par exemple... Pour des pasteurs, quand ils lisent ça, ça leur parle tout de suite, c'est exactement la vie des pasteurs tibétains ('brog pa'i 'tsho ba da ga rang red).

9 Être « écrit en kha skad » indique une proximité relative. Joies et peines sont plus proches de l'oralité que les textes auxquels le comparent implicitement les lecteurs lorsqu'ils le qualifient ainsi. Ni les auteurs classiques ni les contemporains ne mettent par écrit la langue que l'on parle «telle quelle ", de façon aussi systématique que Nülo, c'est-à-dire avec non seulement son vocabulaire et son registre familier, souvent régional, mais aussi ses formes verbales, sa phonologie et ses structures syntaxiques particulières. 
Nülo met par écrit des mots «qui n'ont pas d'orthographe»: une langue qui généralement n'entre pas dans le domaine de l'écrit et dont les mots, de ce fait, ne possèdent pas de transcription standardisée. Le « récit de paroles », dans Joies et peines, fait ainsi l'objet d'un traitement particulièrement réaliste au regard de son traitement habituel dans les œuvres littéraires tibétaines, y compris les ouvrages de fiction contemporains, où, en dépit de l'éventuelle présence de vocables relevant du parler oral (Cham Tshang, 2013), la langue demeure pour l'essentiel régie par les conventions du tibétain littéraire.

L'ouvrage de Nülo entremêle les registres oral et écrit qui peuvent être considérés, dans une perspective linguistique, comme deux pôles. Dans Joies et peines, les dialogues transcrivent assez fidèlement la manière dont le tibétain est parlé, les titres de chapitre et un court passage de prières sont en vers, tandis que le reste du texte est en prose. L'orthographe est quelque peu instable, mais de nombreuses graphies non standard reflètent en réalité la prononciation locale. En dehors du discours direct, l'écriture de Nülo, globalement conforme aux normes de la langue écrite, est cependant caractérisée par l'utilisation d'un langage simple et des césures relativement fréquentes - une ponctuation reflétant davantage les rythmes de l'oralité que les règles de la syntaxe écrite.

11 Loin d'adopter passivement les formes habituelles du tibétain littéraire moderne, Nülo a ainsi pris la liberté d'innover, en mettant par écrit "tels quels» des parlers qui demeurent généralement écartés du registre écrit, en l'occurrence des variétés de dialectes pastoraux de l'Amdo et du Kham. Cet usage, de prime abord quelque peu déconcertant pour un lecteur tibétain, et limitant de fait l'aire de réception de l'ouvrage, donne lieu, parmi les Amdowas, à des expériences de lecture et à des réceptions très contrastées.

Extrait d'un article présentant Joies et peines de l'enfant Naktsang paru dans Latse Library Newsletter :

One issue was that the book was not written in literary language, but in a local, nomadic Amdo Machu County dialect. This affects the readership among Tibetans, as non-Amdowas may experience difficulty understanding local terms and meanings. (Anon, 2008).

15 novembre 2008, Xining. Lurgyal, diplômé d'université, la trentaine, originaire de Yardzi (chi. : Xunhua), préfecture du Haidong (Qinghai), à quatre cents kilomètres de Chukhama: Selon lui Joies et peines n'est pas confortable à lire (mi bde gi) :

C'est parce que c'est du kha skad d'ailleurs, l'auteur est de Golok, ou... Il vient d'un endroit différent. Si c'était en tibétain amdo standard (a mdo spyi skad), ce serait mieux...

Quand je lui demande quel est ce spyi skad de l'Amdo, il cite en exemple celui de la télévision, avant de concéder que c'est une langue souvent proche du littéraire, que les gens du peuple (mi dmangs) ne comprennent pas toujours bien.

17 décembre 2008, canton de Windo (district de Yardzi), à quatre cents kilomètres de Chukhama. Une lycéenne locale :

Ce n'est pas très facile à lire, à cause du kha skad, il y a des mots que je ne comprends pas. (...) Le littéraire est plus facile à comprendre (go kha rtsa gi). Les Contes facétieux du cadavre, les écrits de Dondrubgyäl,... ça, c'est facile à lire.

Dans l'éventail des dialectes amdo, les parlers des agriculteurs de Yardzi sont assez éloignés des dialectes pastoraux représentés dans le livre, qu'il s'agisse de ceux de Machu, Golok ou Chumarlep 5 . Si les linguistes ont invalidé le mythe d'une langue pastorale ('brog skad) commune à l'ensemble des éleveurs-pasteurs tibétophones, il est pertinent de distinguer, à l'échelle de l'Amdo, deux grandes variétés de tibétain: le 
parler des agriculteurs (rong ba), appelé rong skad, et celui des pasteurs ('brog pa), qu'on appelle 'brog skad. La langue de Joies et peines est en ce sens souvent qualifiée de «langue pastorale ». Bien qu'il regrette que Nülo n'ait pas écrit dans un tibétain de l'Amdo que tout le monde puisse comprendre, en langue « commune » ou « standard » (spyi skad) de l'Amdo, Lurgyal reconnaît qu'une telle langue est en pratique difficile à localiser.

13 L'incompréhension de certains mots est une situation qui peut aussi se produire dans le contexte d'interlocution orale de la vie courante, sans poser pour autant de problème majeur. Dans les grands monastères, dans les institutions scolaires, en milieu urbain ou lors de migrations de travail saisonnières, des Amdowas originaires de régions différentes se côtoient et communiquent, de manière le plus souvent efficace. On peut donc se demander dans quelle mesure le kha skad écrit par Nülo constitue un obstacle à la compréhension du texte. Situation de lecture et situation de vie courante ne sont cependant pas identiques. Dans une situation d'échange en face à face, les possibilités d'interaction permettent l'accès à d'éventuelles explicitations. Dans la situation de lecture, en revanche, le lecteur se trouve seul face au texte. Il peut cependant, comme dans la vie courante, s'appuyer sur le contexte des expressions ou des termes incompris pour saisir le sens global du propos. En l'absence de données géographiques précises quant à l'aire de réception de l'ouvrage, on peut supposer, vu son volume de diffusion, que le texte reste globalement lisible pour des locuteurs de différentes variétés de tibétain amdo.

20 octobre 2009, Xining, un étudiant, 25 ans, rong ma 'brog (" ni champ ni pâtures », c'est-àdire pratiquant à la fois l'agriculture et l'élevage) originaire du Tsolho (chi.: Hainan, Qinghai), à cinq cents kilomètres de Chukhama:

Avant, j'avais juste lu quelques bouts par-ci par-là, je pensais que... Mais en lisant le livre, en fait, je comprends tout. Il est de l'Amdo, non? Je me suis rendu compte que chez moi (yul nas) les gens parlent exactement comme ça...

9 février 2009, Xining, femme d'environ 40 ans, originaire comme Nülo de la région de Machu (chi. : Maqu) dans la PAT du Kanlho :

C'est vraiment du parler de Machu, c'est très confortable à lire. Mais même moi, parfois, je ne comprends pas tout. Je suis allée vivre au chef-lieu de district quand j'étais encore très jeune... Les gens du bureau ont souvent des problèmes de compréhension, avec ce texte. Pour les rong $b a$, c'est difficile, il y a des différences, entre la langue qu'ils parlent et le 'brog skad, et même au sein du 'brog skad, il y a parfois des différences entre les langues parlées dans des vallées très proches les unes des autres...

26 mai 2009, Paris, entretien avec Noyontsang Lhamokyap :

[Joies et peines] c'est écrit surtout en langue ordinaire (phal skad), c'est la langue pastorale de son propre pays natal. (...) À mon avis, sa manière d'écrire, elle est jolie (snying rje po), habile (mkhas po). (...) [Dans] le discours direct, c'est la langue locale de chez lui (kho rang gi yul skad). En plus d'être en dialecte (yul skad), dans les dialogues il y a un art de la parole particulier. C'est de la langue ordinaire (phal skad). Mais ce n'est pas seulement de la langue ordinaire, c'est quelqu'un qui connaît aussi, au sein de la langue ordinaire, les caractéristiques d'un certain art de la parole. Cette langue, c'est une langue ordinaire dotée de qualités artistiques.

L'évaluation de l'écriture de Nülo ne tient pas à la seule compréhension littérale du texte. Certes, on l'évalue, pour soi ou pour les autres. On juge aussi du confort de lecture, on relève l'irritation ou le plaisir qu'elle suscite, on l'apprécie d'un point de vue littéraire. Les usages dialectaux peuvent aussi revêtir un caractère ludique, faisant fréquemment l'objet d'imitations amusées. La rencontre de la langue parlée, fut-ce par la médiation de l'écrit, conduit à se situer différemment par rapport à celle-ci, selon qu'on la reconnaisse comme plus ou moins proche de sa propre langue maternelle. Il 
s'agit ici d'observer des actes d'identification et des rapprochements, des mises en relation opérées dans des situations concrètes, non des identités qui se réduiraient à de simples catégories d'appartenance, abstraites et figées (Cottereau et Marzok, 2012, 325-327). La proximité est un rapport dynamique, et il est crucial pour l'enquête d'observer ces différentes manières de "se rendre proche » et de "se sentir proche " (Ricœur, 2000, 162). L'étudiant cité ci-dessus (20 octobre 2009), par exemple, avait toujours insisté sur le fait que le kha skad mis par écrit était pour lui très gênant. Lorsqu'il s'est décidé à lire Joies et peines, il dit s'y être très vite habitué, finissant par trouver la langue écrite par Nülo tout à fait familière, au point de dire : « chez moi (yul nas) les gens parlent exactement comme ça ».

La pluralité des appréciations et des manières d'identifier les dialectes est liée à plusieurs facteurs. D'une part le degré de familiarité effectif avec les usages dialectaux propres à différentes variétés de tibétain, qui est influencé par le lieu d'origine, la biographie, les fréquentations, la formation de chaque lecteur. Les langues écrites par Nülo sont ainsi reconnues comme étant du 'brog skad (langue pastorale), ou plus précisément comme du parler de Golok, de Machu, de Khyokho. D'autre part les critères d'ordres divers qui sont mobilisés pour rattacher un usage particulier à des formes plus générales, qu'il s'agisse de registres de langue ou de différentes échelles géographiques: phal skad et kha skad des langues ordinaire et parlée(s), bod yig du tibétain écrit, yul skad et lung skad des langues locales, am skad de la grande région linguistique du nord-est tibétain. Ces différentes identifications ne sont pas mutuellement exclusives, mais reliées les unes aux autres, souvent enchevêtrées et parfois impliquées les unes dans les autres. Identifier la langue de l'autre, c'est ainsi, dans un même mouvement, qualifier sa propre langue, et les situer toutes deux dans l'ensemble à la fois englobant et divers du tibétain (bod skad), auquel l'appartenance demeure évidente, commune et incontestée.

\title{
Trouver les mots justes, pour (se) rapprocher de l'expérience vécue
}

\author{
24 décembre 2008, Xining, un professeur d'université, environ 35 ans, originaire du Malho, à \\ quelques quatre cents kilomètres de Chukhama: \\ La raison pour laquelle il a écrit ainsi, ça, il faudrait le lui demander... On peut \\ envisager différentes raisons. Peut-être que lui-même ne connaît pas très bien le \\ tibétain écrit, alors peut-être que pour lui c'était plus facile d'écrire comme ça. Mais \\ il aurait pu se faire corriger par quelqu'un... Alors il y a peut-être une autre raison. \\ En tibétain, l'oral et l'écrit sont très éloignés. À la base, l'écriture, c'est l'affaire des \\ monastères, c'est l'écriture des textes sacrés. Peut-être qu'il a voulu rapprocher \\ l'écriture du peuple. » Il me montre en manipulant des objets posés sur la table: \\ «Voilà le monastère, voilà l'écriture ; le peuple est là ; maintenant l'écriture est là \\ [à mi-chemin du peuple et du monastère]. Alors peut-être l'auteur a-t-il voulu la \\ mettre ici [du côté du peuple]... »
}

Ce professeur évoque non plus la relative proximité linguistique entre divers dialectes parlés, mais la distance séparant différents groupes sociaux de l'écrit. L'éloignement qui est évoqué entre le peuple et l'écriture n'est cependant nullement synonyme d'absence de familiarité avec l'écrit. Le rapport à l'écrit, dans toutes les couches de la société, est au contraire imprégné d'usages et de conceptions partagés à l'échelle de l'aire culturelle tibétaine et liés au caractère sacralisé de l'écriture, qui est avant tout chos skad, « langue du dharma » (Tournadre, 2005 ; Diemberger, 2012). 
4 février 2009, Xining, un homme, la trentaine, 'brog pa, originaire de Machen PAT de Golok (chi. : Guoluo, Qinghai), à quatre cents kilomètres de Chukhama :

C'est très agréable et facile à lire, ce livre, c'est comme si quelqu'un parlait. Quand on lit, souvent, il faut s'arrêter pour réfléchir, c'est compliqué, fatigant, ça donne un peu envie de dormir. Mais ce livre-là, ce n'est pas du tout comme ça, c'est comme si quelqu'un te parlait, c'est très agréable (skyid po) à lire.

Les évaluations considérées jusqu'à présent émanaient surtout de personnes habituées à manier le tibétain littéraire. Lorsqu'elles jugent qu'un texte rédigé en langue littéraire est plus compréhensible, elles envisagent une aire de réception plus étendue, non entravée par la diversité dialectale, et une communauté de lecteurs virtuellement composée par les lettrés de l'ensemble de l'aire linguistique tibétaine. Le professeur cité (24 décembre 2008), quant à lui, considère une aire de réception pour ainsi dire plus dense, étendue, au sein d'une même région linguistique, aux lecteurs alphabétisés, mais maîtrisant mal le tibétain littéraire. « Les gens éduqués peuvent le lire, et aussi les gens qui n'ont pas beaucoup d'éducation ", dira-t-il dans la suite de notre entretien. Très éloigné de la langue parlée ordinaire, avec ses phrases longues aux structures syntaxiques complexes et ses formes verbales spécifiques, le tibétain littéraire demeure en effet relativement hermétique à ceux qui n'en sont pas familiers ${ }^{6}$.

L'accessibilité de son écrit aux lecteurs "populaires », aux « simples "brog pa », comme le devine ce lecteur (24 décembre 2008), est en effet l'une des aspirations de Naktsang Nülo lorsqu'il élabore son texte ${ }^{7}$. S'il évoque aussi son manque de compétence en tibétain littéraire et affirme être plus à l'aise en chinois, Nülo, pour qui écrire en tibétain s'est imposé comme une nécessité, souligne d'emblée avantages du kha skad. Bien plus qu'une solution par défaut, l'écriture en kha skad se présente ainsi comme résultant d'un choix délibéré.

9 mars 2009, Xining, entretien avec Naktsang Nülo:

Au moment d'écrire [Joies et peines], il fallait que j'écrive en tibétain. (...) j'avais cette idée. Si j'écris en tibétain, moi je suis amdo, n'est-ce pas? Dans le Gansu, là, je connais le parler de Khyokho. En mettant par écrit ce parler là, même si je ne sais pas écrire conformément aux [règles grammaticales et orthographiques des deux ouvrages de référence] Sum rtags et Rtags mjug... je me suis dit que ça allait, si j'écrivais en kha skad. (...) Et puis il y a un avantage, à écrire en kha skad. Les savants, ceux qui ont un niveau de culture très élevé, se diront peut-être: "Eh! Il y a quelque chose qui ne va pas, là !» Mais autrement, la plupart des gens ordinaires, si on écrit en kha skad, s'ils l'entendent, de leurs oreilles cela tombe [directement] dans leur cœur. "Ah, ça, c'est exactement notre langue, la langue du pays natal!» Les aînés et les personnes âgées, et maintenant les jeunes, quel que soit l'âge des gens, lorsqu'ils lisent ce livre, ils ressentent de la tristesse, ils sont émus aux larmes. Beaucoup de cela tient au kha skad.

19 Cette dernière "explication » apportée au choix d'écrire en kha skad entrait-elle en considération au moment de l'écriture ou est-elle basée sur les retours des lecteurs après la sortie du livre? Quoi qu'il en soit, aujourd'hui cette faculté à toucher les lecteurs, à les émouvoir, bien souvent aux larmes, constitue un élément très important aux yeux de Nülo, et ce dernier explique par la présence du kha skad la capacité, avérée, de son texte à susciter l'émotion.

21 août 2012, Machu, chez Naktsang Nülo :

Il m'explique avoir revu son manuscrit, après la publication initiale du livre. Il a rétabli le kha skad là où, par automatisme d'écriture, des graphies ou des termes littéraires plus standards s'étaient glissés dans le texte. Dans la version définitive, il a rétabli des graphies et des termes conformes aux usages locaux: yed au lieu de byed (« faire »), 'gyo au lieu de 'gro (« aller »), ba pour thub (« pouvoir »), zha yas pour 
byis pa (« enfant »)... Dans mon édition, qui est celle d'une version antérieure, il m'explique en lisant dans le texte, en me montrant des occurrences et en les commentant. "Les gens ne disent pas ça, ici on dit comme ceci », « Ici, c'est écrit "da ngas cang ma thub tha" ("J'ai rien pu faire »), on ne dit pas ça, en kha skad on dit ma ba tha. » En me montrant les dernières phrases de son père, au seuil de la mort, confiant ses deux enfants à ses camarades, il remarque "byis pa, c'est du yig skad ", puis relit à nouveau la phrase en remplaçant ce mot par zha yas. « Là, c'est vraiment comme ça », remarque-t-il, plus satisfait.

$\mathrm{Au}$ fil d'entretiens avec Naktsang Nülo, j'ai progressivement commencé à saisir que l'écriture "en kha skad » répondait également (ou peut-être avant tout?) au vœu de représenter fidèlement des paroles inéluctablement à jamais perdues, dans leur oralité, dans leur énonciation première. Mais des paroles qu'il fallait à présent représenter, recréer par et dans l'écriture, de manière à les représenter fidèlement, à les faire résonner aujourd'hui, sans qu'elles ne sonnent faux.

\title{
Un accent d'authenticité
}

\begin{abstract}
5 décembre 2008, Xining, un lama d'environ 35 ans originaire de Golok, à environ deux cent cinquante kilomètres de Chukhama:

Il se plonge dans le livre, lit un peu, assez lentement, puis, très animé, souriant, il s'exclame : «Ce sont de vraies paroles (skad cha ngo ma red)! C'est notre parler (ngi cha'o gi skad cha red)!

26 mai 2009, Paris, entretien avec Noyontsang Lhamokyap :

[La] manière de parler de nos pères, et des pères de nos pères, la manière de parler des gens d'avant, la vraie manière de parler propre aux 'brog pa, nous ne la connaissons pas. (...) Quand on lit ça, on se rend compte que les écrivains, nous avons des insuffisances, des points faibles. (...) Sa langue à lui, c'est vraiment une langue ordinaire authentique (yang dag), pure (slad med). Pour moi, en tant que lecteur, sa manière d'écrire et de mener le récit, cette manière de parler, c'est de l'art. (...) C'est l'art du parler populaire, oral, qui ne s'embarrasse pas de vérifier si les mots sont dans le dictionnaire ou non. Pourquoi les écrivains contemporains ne sont-ils pas à même d'écrire ainsi ? Tu pioches quelques termes mélodieux dans les dictionnaires et [tu empruntes] à l'art de la métaphore, tu arranges et réarranges [ton texte], en rapportant quelques proverbes, tu les places ici et là. Mais en réalité, notre véritable langue de 'brog $p a$, tu ne la parles pas.
\end{abstract}

21 Amdowa, 'brog pa, en exil depuis près de vingt-cinq ans, écrivain et poète, enseignant et excellent connaisseur de la littérature tibétaine, Lhamokyap dessine une vision complexe de la langue commune, laissant entrevoir la nature des partages et des chevauchements constitutifs du système écolinguistique tibétain. Si dans d'autres situations il insistera sur l'unité de la nation tibétaine, y compris au niveau de sa langue, il décrit ici une situation où les parlers, plus ou moins partagés, diffèrent entre localités, mais aussi entre générations, selon les parcours de vie et les environnements fréquentés plus ou moins durablement par les uns et les autres.

Il faut noter que c'est le fait qu'elle soit mise par écrit qui permet à Lhamokyap de prendre cette distance avec la langue, qui lui permet de s'y arrêter, pour la comparer à d'autres écrits et à d'autres parlers. Face à la spontanéité, à l'éloquence innée du parler populaire, «qui ne s'embarrasse pas de vérifier si les mots sont dans le dictionnaire ou non » avant de s'exprimer, le style des écrivains contemporains apparaît tout d'un coup un peu forcé, comme manquant d'authenticité, déconnecté du registre de l'oralité populaire. 
26 mai 2009, Paris, Noyontsang Lhamokyap :

Mon père, moi, ceux qui sont plus jeunes que moi, notre manière de parler diffère complètement. Même l'intonation est différente. a bhe lo lo, khyod thon btang ne... ( A bhe lo lo, te voilà, toi?»). Par exemple les vieux, avant, pour exprimer de l'affection pour un jeune enfant - [ils disaient] $U$, 'di da zhi lu ya rabs zig yin, kha nyan ni red («Ouh! C'est mon garçon bien sage, ça! Bien obéissant!»). [Ils emploient] des manières de dire plus longues, ou plus courtes... Il y a quelque chose. Les gens comme nous, maintenant, on ne parle pas comme ça. [On dit:] bu gu pe yag po 'dug, zha yas yag po zig red ("Il est très mignon, ce petit », en tibétain du Ü-tsang et de l'Amdo) - on dit ça, mais l'intonation, les petits mots qui n'ont pas de sens particulier, les proverbes, rien de cela ne nous vient.

9 novembre 2011, Dharamsala. Dorjé, 'brog pa originaire du Tsolho, la vingtaine, travaille à la bibliothèque :

Il a lu Joies et peines en une semaine. "C'est hallucinant, c'est hallucinant comme c'est bien écrit! (ha las ba bris yod gi, ha las ba yag ga bris yod gi) », répète-t-il à plusieurs reprises. "C'est vraiment un livre à lire. Surtout avec l'écriture en tibétain de l'Amdo, c'est vraiment la manière dont parlent les vieux, les gens du pays. Il arrive à faire éprouver des ressentis, il parvient à émouvoir (tshor ba ster thub gi, sems 'gul thebs thub gi). On voit vraiment comment les choses se sont déroulées.

La langue vernaculaire dans Joies et peines a ainsi ceci de particulier qu'elle constitue, pour de nombreux lecteurs, une langue que l'on n'entend plus - c'est notamment le cas pour des Amdowas originaires de régions pastorales, mais installés en ville, au Tibet, et, davantage encore, pour les Amdowas vivant en exil. Dans la diaspora tibétaine, la langue véhiculaire est proche du tibétain de l'Ü-tsang. Arrivés en exil beaucoup plus récemment, pour la plupart depuis les années 1980, demeurant une petite minorité au sein de la diaspora, les Amdowas ont dû apprendre "sur le tas ", parfois à grand-peine, le tibétain parlé de l'exil. Dans ce processus, quelques-uns développent une langue hybride, mêlant tibétain amdo et spyi skad (« langue commune ») de l'exil, rencontrant ensuite des difficultés pour communiquer avec leurs proches restés au pays. Pour certains, cette langue - avec ses tournures de phrase, ses proverbes et ses jurons caractéristiques des 'brog $p a$ - dont la lecture amuse et émeut, résonne sans doute des sonorités de l'enfance et rappelle le pays natal bien-aimé laissé derrière soi, rendant plus vif encore le caractère douloureux de l'exil.

\section{"Faire éprouver des ressentis »}

Janvier 2009, Xining, une étudiante:

Quand je lisais ce livre, c'était comme si j'étais moi-même quelqu'un de l'époque de la Révolution culturelle ${ }^{8}$,je ressentais une grande frayeur, comme si les événements décrits en détail dans le livre se passaient juste à côté de moi.

Nombreux sont les lecteurs qui disent avoir l'impression, en lisant, d'écouter « un vieux grand-père " raconter une histoire, de "regarder un film», d'« entendre" les dialogues, de « voir » les situations décrites, et même parfois de "sentir » les odeurs. En inscrivant la langue parlée dans son récit, Nülo parvient ainsi à rendre l'expérience de l'enfant à la fois très présente et très proche. L'action, par une lecture sans effort, se déroule d'elle-même devant les yeux du lecteur, comme une expérience de découverte partagée avec l'enfant. Les dialogues, rédigés en langue parlée, constituent comme un commentaire en direct des événements, exprimant des évaluations et des compréhensions en cours d'action. Il ne s'agit pas de dire ici que l'écriture «imite » ou "reproduit» le réel ${ }^{9}$, mais de souligner que dans ce récit que Nülo rédige presque 
cinquante ans après les faits, il se donne pour but de rendre compte de son expérience sans y superposer de compréhension ou de mise en forme ex post, y compris en ce qui concerne la langue parlée ${ }^{10}$. Le sentiment d'immédiateté mis en avant par de nombreux lecteurs, créé par l'utilisation de la langue vernaculaire associée à un récit strictement focalisé sur l'enfant, suggère, pour décrire ces expériences de lecture, la notion d'une mimèsis narrativeréussie.

Bousculant les traditions littéraires dominantes, en mettant par écrit la langue parlée entre proches, dans son registre le plus simple, le plus familier, le plus intime, Joies et peines sont connectées à des registres relevant généralement, dans le contexte tibétain, plutôt de l'oralité. Mokchung Purkho, un écrivain amdowa, souligne combien la langue parlée maternelle, par opposition ici non à une langue étrangère, mais à la langue écrite, est associée au registre de l'affect :

Si l'on compare les langues locales et vernaculaires (lung skad dang phal skad) à la langue littéraire (yig skad) - la langue commune tibétaine (bod kyi spyi skad) - régie par les règles de la science grammaticale, les différences, tant dans la prononciation que dans la graphie, sont certes assez nombreuses, mais tout le monde sait que notre affection est plus grande pour [la langue] que nous ont enseignée nos parents que pour la langue écrite. (Mog chung, 2010, 203)

En dépit des multiples formes de distances - dialectales, spatiales, générationnelles - et parfois en raison même de celles-ci, la lecture des langues parlées mises par écrit dans Joies et peines crée un sentiment d'authenticité, des expériences d'immédiateté et des rapprochements. La lecture du texte donne ainsi lieu à une forme particulière de partage de l'expérience passée, rendue présente par la double médiation d'un texte écrit et d'un acte de lecture.

Isabelle Henrion-Dourcy, dans son analyse des récits produits par des demandeurs d'asile tibétains en Belgique, suggère l'existence d'un «frein culturel » à l'exposition des souffrances. Cette réticence est due, selon elle, non tant à la pudeur qu'à des " conceptions relatives à la manière 'interdépendante' dont s'enchaînent les phénomènes (tendrel, concept d'origine bouddhiste qui a trouvé un large écho dans la pratique sociale). Il ne porte pas chance de raviver la mémoire des traumatismes passés, car cela leur redonne un pouvoir d'action sur le présent " (Henrion-Dourcy, 2007, 138). L'existence supposée d'un « frein culturel» à l'exposition des souffrances impliquerait-elle, en miroir, des freins à la réception de leurs récits? À la lumière de l'enquête, il semblerait que pour évoquer Cinquante-huit, Naktsang Nülo soit parvenu à trouver, aux yeux de plusieurs milliers de lecteurs, des mots qui sonnent juste.

\section{BIBLIOGRAPHIE}

ANON, 2007-2008, An Introduction to The Joys \& Sorrows of a Boy from Naktsang (Naktsang Shilu Kyiduk), Latse Library Newsletter, vol. 5, pp. 54-58.

CHAM TSHANG PADMA LHUN GRUB, 2013, Yig skad dang kha skad kyi khyad par dang de'i bstun thabs skor la dpyad pa [Analyse des différences entre langue écrite et langue parlée, ainsi que des manières de 
les réconcilier], Papier présenté lors de la $3^{\text {rd }}$ Conference on Tibetan Language (New York, 9-14 décembre 2011), mis en ligne le 01/11/2013, http://blog.amdotibet.cn/padmalhundrup/ index.aspx, consulté le 24/02/2014.

COTTEREAU, Alain, MARZOK, Mokhtar Mohatar, 2012, Une famille andalouse : ethnocomptabilité d'une économie invisible, Paris, Bouchène.

DIEMBERGER, Hildegard, 2012, Quand le livre devient relique : les textes tibétains entre culture bouddhique et transformations technologiques, Terrain, $n^{\circ}$ 59, p. 18-39.

FAVRET-SAADA, Jeanne, 1990, Être affecté, Gradhiva, nº 8, p. 3-10.

GENETTE, Gérard, 1972, Figures III, Paris, Seuil.

GREEN, Jeffrey R., 2012, Amdo Tibetan Media Intelligibility, SIL Electronic Survey Reports 2012-019, http://www.sil.org/resources/publications/entry/48318, consulté le 06/03/2014.

HENRION-DOURCY, Isabelle, 2007, De l'exil à l'asile : témoignages et authenticité culturelle des candidats tibétains au statut de réfugié politique en Belgique, Civilisations, n ${ }^{\circ}$ 6, p. 121-158. HEERING, Xénia de, 2012, les Pratiques de lecture dans l'Amdo contemporain, Monde chinois, nouvelle Asie, $\mathrm{n}^{\circ} 31$, p. 64-70.

HEERING, Xénia de, 2014, Breaching and Bridging Literary Traditions? A few observations on a text written in « kha skad » and its translation into literary Tibetan, in Pavel L. Grokhovskiy (ed.), Modernizing the Tibetan Literary Tradition. Abstracts and Papers $\left[6^{\text {th }}\right.$ International scientific conference, "Issues of Far Eastern Literatures", June 25-29, 2014], Saint-Pétersbourg, NP-Print, pp. 8-16.

JURGENSON, Luba, 2003, l'Expérience concentrationnaire est-elle indicible?, Monaco,

Éditions du Rocher.

KLU SMYON HE RU KA, 2012, Ma bklags tshad med kyi dpe deb bklags pa [Un livre à lire absolument : lecture], publié le 13/01/2012, Khabdha, http://www.khabdha.org/?p=24421, consulté le 05/05/2014.

MOG CHUNG PHUR KHO, 2010, Pha mas bdag la 'di skad gsungs: a mdo'i mkhas dbang dang a mdo'i phal skad [les Paroles que m'ont adressées mes père et mère : les érudits amdowas et le parler ordinaire de l'Amdo], Beijing, Mi rigs dpe skrun khang.

NACANG NULUO, 2011, Na nian, shishi fanzhuan: yi ge xizangren de tongnian huiyi [Ces années-là, le renversement d'époque. Souvenirs d'enfance d'un Tibétain], Taïwan, Xueyu.

NAGS TSHANG NUS BLO, 2007, Nags tshang zhi lu'i skyid sdug [Joies et peines de l'enfant Naktsang], Xining, Qinghai Xining Yinshuachang.

NAGS TSHANG NUS BLO, 2008, Nags tshang zhi lu'i skyid sdug [Joies et peines de l'enfant Naktsang], Dharamsala, Kha ba dkar po bod kyi rig gzhung sri zhu khang.

RICÆUR, Paul, 2000, la Mémoire, l'histoire, l'oubli, Paris, Seuil.

ROBIN, Françoise, 2012, la Révolte en Amdo en 1958, l'histoire du Tibet du XVII ${ }^{e}$ au XXI ${ }^{e}$ siècle : compte rendu de la journée de conférences au Sénat le 3 mars 2012, Rapport de groupe interparlementaire d'amitié ${ }^{\circ}$ 104, publié le 18/06/2012, http://www.senat.fr/ga/ga104/ga104.html, consulté le 08/02/2014.

SEMPRUN, Jorge, 1994, l'Écriture ou la Vie, Paris, Gallimard. 
TOURNADRE, Nicolas, 2005, L'Aire linguistique tibétaine et ses divers dialectes, in Daniel Petit (éd.), Lalies 25, Paris, Éd. Rue d'Ulm, p. 7-56.

\section{ANNEXES}

\section{Extrait de Joies et peines de l'enfant Naktsang (5 partie, chapitre 68)}

À l'automne 1958, des troupes de l'Armée populaire de libération arrivent à Chukhama. Naktsang Durkho décide de prendre la fuite, dans l'espoir d'échapper aux violences qui touchent alors l'Amdo. Emmenant ses deux fils - Nülo, dix ans, et Japé, quatorze ans et rejoint par quelques compagnons, il guide le groupe vers le Tibet central. Moins de deux mois plus tard, deux jeunes moines et Durkho seront tués dans une embuscade de l'APL. Les neuf survivants se rendent alors aux mains des soldats. Les deux garçons Naktsang, refusant d'être séparés de leurs compagnons, sont inclus dans un convoi de prisonniers qui prend, sous escorte militaire, la route de la prison du district de Chumarlep. La scène qui suit se déroule lors d'une escale, au lendemain de leur arrivée dans un camp-prison où sont retenues au total près de cent cinquante personnes.

Dans la tente, les prisonniers épanchaient leurs douleurs, évoquant chacun les familles et les foyers qu'ils avaient laissés. Oncle Tendzin et Lochö ont beaucoup discuté de comment on s'était battus avec les soldats chinois et de comment ils avaient tué des Chinois. Comme j'avais un peu faim, j'ai mis la main dans ma poche et dans mon bol j'ai pris un peu de tsampa ${ }^{1}$, et puis je l'ai donnée à Grand frère Japé. « J'en ai encore, mange, toi! Mais t'en mange qu'un tout petit peu, hein !» m'a-t-il dit. Je n'avais pas encore touché à la tsampa que la femme d'hier avait mise dans mon bol. Comme Grand frère Japé m'a dit qu'il n'en voulait pas, j'en ai mis dans ma bouche et je l'ai mangée. À ce moment, les gens à côté de moi ont commencé à dire : «Oh, quelle délicieuse odeur de tsampa, tout d'un coup! C'est qui qui a de la tsampa? » Et puis, en tendant la main, un vieillard à la tête blanche m'a demandé : «Eh, toi, gamin ! Tu me donnerais un peu de tsampa? » Quand j'ai vu un vieillard comme ça tendre la main vers moi, je me suis senti très mal à l'aise. J'ai pris une petite bouchée de tsampa dans mon bol, et je l'ai mise dans sa main. «Oh, longue vie à toi ! Garçon bienveillant ! Ça fait près de vingt jours que, moi, le vieillard, je suis arrivé là, et que j'ai pas senti l'odeur de la tsampa... J'en ai la tête qui tourne, là... » a-t-il dit. Il a un peu frotté la tsampa dans ses mains, et ensuite il l'a frottée sur son cou et sur sa tête. Et puis il en a encore détaché un petit bout, et il l'a donné à un autre vieux, à côté de lui, en lui disant : «Eh, toi ! Frotte-toi le cou et le dos avec ça, ça apaise le lung².» Le petit peu de tsampa qui lui restait encore, il l'a approché de son nez pour en sentir un peu l'odeur, et puis il est resté en le tenant dans sa main. Je me disais : « Le pauvre... Ça peut pas être qu'il veut pas manger, c'est qu'il peut pas se décider à le faire... » (Trad. X. de Heering) 


\section{NOTES}

1. Le tibétain est translittéré en Wylie. Pour les noms propres, une transcription visant à faciliter la lecture aux non-spécialistes a cependant été adoptée. En dehors des références bibliographiques, le nom de l'auteur de Joies et peines est ainsi orthographié Naktsang Nülo, ce dernier étant également désigné par son nom raccourci Nülo. Mes remerciements vont en premier lieu à tous ceux qui, au Tibet, en Inde ou en France, ont bien voulu partager avec moi leurs idées et leurs impressions de lecture. Je tiens en outre à remercier chaleureusement Marie-Paule Hille (CECMC, EHESS), ainsi qu'Eric Mélac (Paris 3), pour leur relecture attentive et leurs suggestions. Toute inexactitude n'est imputable qu'à moi-même.

2. Parfois appelé Domé (Mdo smad), linguistiquement l'Amdo comprend la quasitotalité du Qinghai, ainsi que certaines zones du Gansu et du Sichuan. «Tibet » désigne ici, conformément à l'usage de mes enquêtés, l'ensemble de l'aire de peuplement tibétain, dont les principales régions sont l'Ü-tsang (Tibet central), le Tö-ngari (Tibet occidental), le Kham (Tibet oriental) et l'Amdo (nord-est du Tibet).

3. Il existe en outre deux traductions : en chinois (Nacang, 2011) et en tibétain écrit «standard» (Nags tshang, 2008), cette dernière étant commentée ailleurs (de Heering, 2014). Une version abrégée en anglais, sous le titre My Tibetan Childhood: When Ice Shattered Stone, est à paraître chez Duke University Press.

4. L'enquête a été réalisée principalement dans la province du Qinghai, entre 2008 et 2012. Mes séjours d'enquête au Tibet ont bénéficié du soutien du CEFC, du CECMC et de l'EFEO. À l'automne 2011, une enquête plus limitée a été réalisée à Dharamsala, siège du gouvernement tibétain en exil, qui concentre $15 \%$ des 94000 Tibétains recensés en Inde.

5. L'intercompréhension entre locuteurs de différents dialectes tibétains étant un sujet relativement peu exploré par les linguistes, il est impossible d'évaluer «objectivement » le degré de compréhension potentiel du texte par des Tibétains de telle ou telle localité.

6. Une étude récente (Green, 2012) portant sur la compréhension de la langue utilisée dans les médias audiovisuels en Amdo a montré que la compréhension orale était limitée parmi les Amdowas peu éduqués dès lors que le registre de langue utilisé était littéraire. La population considérée dans l'étude comptait plus de cent hommes et femmes de différents âges, ayant été scolarisés entre zéro et six années.

7. D'autres caractéristiques de Joies et peines peuvent être mises en relation avec cet objectif : au niveau de l'architecture générale du livre, des chapitres courts, dont les titres résument l'action principale; un récit strictement chronologique; des phrases courtes et une langue simple; enfin, un prix accessible (15 RMB, soit 1,75 euros).

8. "Révolution culturelle" ne désigne pas ici la période 1966-1976 mais Cinquantehuit, un usage relativement fréquent en Amdo, d'après mes observations.

9. Comme le rappelle Gérard Genette, «aucun récit ne peut 'montrer' ou 'imiter' l'histoire qu'il raconte. Il ne peut que la raconter de façon détaillée, précise, 'vivante', et donner par là plus ou moins l'illusion de mimèsis qui est la seule mimèsis narrative, pour cette raison unique et suffisante que la narration, orale ou écrite, est un fait de langage, et que le langage signifie sans imiter » (Genette, 1972, 185). 
10. On peut rapprocher la posture de Nülo du refus explicite du littéraire énoncé par Varlam Chalamov et Primo Levi, que Luba Jurgenson analyse comme "un souci d'authenticité, un refus du romanesque, de la transmutation du réel : le réel doit entrer dans l'œuvre tel quel, saisi dans son authenticité première, dans la pureté absolue de l'image ». (Jurgenson, 2003, 25).

1. Orge grillée moulue, qui se mange le plus souvent pétrie avec de l'eau ou du thé. La tsampa est une denrée de base à travers toute l'aire de culture tibétaine.

2. Énergie (sanscrit : prana). Selon la médecine tibétaine, le lung est une des trois humeurs du corps et sa perturbation est à l'origine de nombreux maux, tant physiques que psychiques.

\section{RÉSUMÉS}

Publiées à Xining (Chine) en 2007, Joies et peines de l'enfant Naktsang, un témoignage historique sans précédent sur les années 1950 en Amdo (nord-est du Tibet), sont très vite devenues un bestseller du marché littéraire local. Contrairement aux pratiques dominant l'écriture du tibétain, le texte entremêle les registres littéraire et oral, mettant les dialectes pastoraux par écrit «tels quels». L'ethnographie des expériences de lecture et des réceptions de l'ouvrage permet d'analyser la diversité des appréciations formulées à l'égard de cette écriture atypique, pour dégager certaines des sources de la justesse de ton qu'un grand nombre de lecteurs reconnaît à l'enfant Naktsang.

Published in Xining (China) in 2007, Joys and Sorrows of the Naktsang Boy, a unique historical testimony about the 1950s in Amdo (north-eastern Tibet), quickly became a best-seller on the local literary market. Contrary to dominant Tibetan writing practices, the book intertwines the oral and the literary registers, writing down pastoral dialects "as they are spoken". Based on an ethnographic study of reading experiences and receptions of Joys and Sorrows, the article analyses the varied assessments of this unusual writing style, while seeking to locate some of the sources of the aptness and justness of tone that many readers recognise to its author.

\section{INDEX}

Mots-clés : expérience de lecture, usages de l'écrit, dialectes, témoignage

Index géographique : Tibet

Keywords : Reading Experience, Amdo (Tibet), Writing Practices, Dialects, Testimony

\section{AUTEUR}

\section{XÉNIA DE HEERING}

Centre d'études sur la Chine moderne et contemporaine, (EHESS), ASIEs, INALCO 